\title{
Organ system-involvement in SLE and relationship with demographic factors, disease duration and health-related quality of life in childhood SLE
}

\author{
Lakshmi N Moorthy ${ }^{14^{*}}$, Maria J Baratelli ${ }^{10}$, Margaret GE Peterson ${ }^{3}$, Afton L Hassett ${ }^{15}$, Alexa B Adams ${ }^{3}$, \\ Laura V Barinstein ${ }^{5}$, Emma J MacDermott ${ }^{3}$, Elizabeth C Chalom ${ }^{8}$, Karen Onel ${ }^{12}$, Linda I Ray ${ }^{16}$, Jorge Lopez-Benitez ${ }^{9}$, \\ Christina Pelajo ${ }^{9}$, Kathleen A Haines ${ }^{2}$, Daniel J Kingsbury ${ }^{4}$, Victoria W Cartwright ${ }^{4}$, Philip J Hashkes ${ }^{7}$, Nora G Singer ${ }^{6}$, \\ Gina A Montealegres ${ }^{11}$, Ingrid Tomanova-Soltys ${ }^{11}$, Andreas O Reiff' ${ }^{1}$, Sandy D Hong ${ }^{13}$, Thomas JA Lehman ${ }^{3}$
}

From 2011 Pediatric Rheumatology Symposium sponsored by the American College of Rheumatology Miami, FL, USA. 2-5 June 2011

\section{Purpose}

Damage in childhood systemic lupus erythematosus (SLE) affects ocular, musculoskeletal, neuropsychiatric, renal, cardiovascular, peripheral vascular, and skin domains and damage can affect their health related quality of life (HRQOL). Aggressive treatments have improved survival in childhood SLE, but the disease is still associated with significant morbidity. The objective of our multicenter study is to examine the organ system-involvement in childhood SLE and the relationship of damage with HRQOL, age, gender, ethnicity and disease duration.

\section{Methods}

In this cross-sectional study, children $\leq 18$ years with SLE and parents completed the Simple Measure of the Impact of Lupus Erythematosus in Youngsters $\odot$ (SMILEY๑) and physicians measured Systemic Lupus International Collaborating Clinics/ACR Damage Index (SDI). SMILEY@ is a new, brief, 24-item HRQOL assessment tool for pediatric SLE, that has recently been validated in US English. The four domains are: Effect on self, Limitations, Social and Burden of SLE. Responses are in the form of a 5-faces scale for easy comprehension. Higher percentage scores indicate better HRQOL. Contingent upon the data distribution of the above variables, we used student t-test, Mann-Whitney U test or the Kruskal-Wallis (KW) test to

\footnotetext{
${ }^{14}$ University of Medicine and Dentistry of NJ (UMDNJ)- Robert Wood Johnson Medical School, New Brunswick, NJ, USA

Full list of author information is available at the end of the article
}

examine the relationship of SDI with age, gender, ethnicity, disease duration and HRQOL.

\section{Results}

Out of a total of 169 children (17\% male), 59 children (35\%) had any damage (SDI score $>0$ ). Their age, damage and disease duration and specific organ-system involvement is given in table 1 . Their ethnicities were: Black (39\%), Asian or Pacific Islander (12\%), Latino (27\%), White (18\%) and other (5\%). Children predominantly had renal, neuropsychiatric, skin, and musculoskeletal involvement. Significant difference was found in damage with disease duration ( $\mathrm{p}=0.005$, Mann Whitney $U$ test). There was no significant difference in damage in patients with different gender, ages or ethnicity. Parent SMILEY@ total and all domain scores were decreased in patients with damage compared to patients without damage (table 2), but only the Effect on self domain scores was statistically significant $(\mathrm{p}=0.02)$. The child SMILEY@ total, Limitation and Effect on self domain scores were lower in patients who had any damage.

\section{Conclusion}

Damage in childhood SLE is significantly related to disease duration. Renal, neuropsychiatric, skin, and musculoskeletal systems are predominantly involved in our US cohort, which is similar to previous studies. The impact of damage on children's HRQOL as perceived by parents 
Table 1 SDI scores and organ-system involvement, and HRQOL scores

\begin{tabular}{|c|c|}
\hline Variables & Descriptives $(n=169)$ \\
\hline \multicolumn{2}{|l|}{ SDI total score } \\
\hline Mean \pm SD (range)(n) & $36 \pm 39(1-183)$ \\
\hline Median & Median $=24$ \\
\hline Age (years) & $15 \pm 3(6-18)$ \\
\hline \multicolumn{2}{|l|}{ Disease duration (months) } \\
\hline Mean \pm SD (range) (n) & $36 \pm 39(1-183)$ \\
\hline Median & Median $=24$ \\
\hline Renal \%(n) & $17(29)$ \\
\hline Neuropsychiatric \%(n) & $14(24)$ \\
\hline Skin $\%(n)$ & $11(18)$ \\
\hline Musculoskeletal \%(n) & $6(10)$ \\
\hline Peripheral vascular \%(n) & $3(5)$ \\
\hline Ocular \%(n) & $2(4)$ \\
\hline Pumonary \%(n) & $2(4)$ \\
\hline Diabetes \%(n) & $2(3)$ \\
\hline Cardiovascular \%(n) & $2(3)$ \\
\hline Gastrointestinal \%(n) & $1(2)$ \\
\hline Premature gonadal failure \%(n) & $1(2)$ \\
\hline Malginancy $\%(n)$ & $0(0)$ \\
\hline \multicolumn{2}{|l|}{ SMILEY total score } \\
\hline Mean \pm SD (range) (n) Child & $65 \pm 14(33-98)(166)$ \\
\hline Parent & $62 \pm 15(28-96)(153)$ \\
\hline
\end{tabular}

may be different from the children's perception and needs further examination.

\section{Disclosure}

Lakshmi N. Moorthy: Arthritis Foundation, 2; Maria J. Baratelli: Arthritis Foundation, 2; Margaret G.E. Peterson: Arthritis Foundation, 2; Afton L. Hassett: Arthritis Foundation, 2; Alexa B. Adams: None; Laura V. Barinstein: None; Emma J. MacDermott: None; Elizabeth C. Chalom: None; Karen Onel: None; Linda I. Ray: None; Jorge Lopez-Benitez: None; Christina Pelajo: None; Kathleen A. Haines: None; Daniel J. Kingsbury: None; Victoria W. Cartwright: None; Philip J. Hashkes: None; Nora G. Singer: None; Gina A. Montealegres: None;

Table 2 Means of parent total and domain scores of SMILEY for patients with no damage $(S D I=0)$ and patients with any damage (SDI>0)

\begin{tabular}{lll}
\hline Parent SMILEY scores & SDI score $=\mathbf{0}(\mathbf{n})$ & SDI score $>\mathbf{0}(\mathbf{n})$ \\
\hline Effect on self domain & $63 \pm 18(96)$ & $57 \pm 16(55)$ \\
Limitation domain & $62 \pm 19(97)$ & $59 \pm 16(55)$ \\
Social domain & $78 \pm 18(97)$ & $74 \pm 17(55)$ \\
Burden of SLE domain & $57 \pm 16(96)$ & $54 \pm 17(55)$ \\
Total score & $64 \pm 15(97)$ & $60 \pm 14(55)$ \\
\hline
\end{tabular}

Ingrid Tomanova-Soltys: None; Andreas O. Reiff: None; Sandy D. Hong: None; Thomas J. A. Lehman: None.

\section{Author details}

${ }^{1}$ Childrens Hospital LA, Los Angeles, CA, USA. ${ }^{2}$ Hackensack University Medical Center, Hackensack, NJ, USA. ${ }^{3}$ Hospital for Special Surgery, New York, NY, USA. ${ }^{4}$ Legacy Emanuel Children's Hospital, Portland, OR, USA. ${ }^{5}$ Maimonides Medical Center, Brooklyn, NY, USA. ${ }^{6}$ MetroHealth Medical Center, Cleveland, OH, USA. 'Shaare Zedek Medical Center, Jerusalem, Israel, USA. ${ }^{8}$ St. Barnabas Medical Center, New Brunswick, NJ, USA. ${ }^{9}$ Tufts Medical Center, Boston, MA, USA. ${ }^{10}$ UMDNJ-Robert Wood Johnson Medical School, New Brunswick, NJ, USA. ${ }^{11}$ University Hospitals Case Medical Center, Cleveland, $\mathrm{OH}$, USA.

${ }^{12}$ University of Chicago, Chicago, IL, USA. ${ }^{13}$ University of lowa Children's Hospital, lowa City, IA, USA. ${ }^{14}$ University of Medicine and Dentistry of NJ (UMDNJ)- Robert Wood Johnson Medical School, New Brunswick, NJ, USA. ${ }^{15}$ University of Michigan Medical School, Ann Arbor, MI, USA. ${ }^{16}$ University of Mississippi Medical Center, Jackson, MS, USA.

Published: 13 July 2012

doi:10.1186/1546-0096-10-S1-A22

Cite this article as: Moorthy et al:: Organ system-involvement in SLE and relationship with demographic factors, disease duration and health-related quality of life in childhood SLE. Pediatric Rheumatology 2012 10(Suppl 1):A22.
Submit your next manuscript to BioMed Central and take full advantage of:

- Convenient online submission

- Thorough peer review

- No space constraints or color figure charges

- Immediate publication on acceptance

- Inclusion in PubMed, CAS, Scopus and Google Scholar

- Research which is freely available for redistribution 